\title{
Localizing Conformational Hinges by NMR: Where Do Hepatitis B Virus Core Proteins Adapt for Capsid Assembly?
}

\section{Journal Article}

Author(s):

Lecoq, Lauriane; Wang, Shishan; Wiegand, Thomas; Bressanelli, Stéphane; Nassal, Michael; Meier, Beat H.; Böckmann, Anja

Publication date:

2018-06

Permanent link:

https://doi.org/10.3929/ethz-b-000271160

Rights / license:

In Copyright - Non-Commercial Use Permitted

Originally published in:

ChemPhysChem 19(11), https://doi.org/10.1002/cphc.201800211

Funding acknowledgement:

159707 - NMR studies in the Solid State (SNF)

741863 - Faster magic-angle spinning leads to a resolution revolution in biological solid-state NMR (EC) 


\section{Accepted Article}

Title: Localizing conformational hinges by NMR: where do HBV core proteins adapt for capsid assembly?

Authors: Lauriane Lecoq, Shishan Wang, Thomas Wiegand, Stéphane Bressanelli, Michael Nassal, Beat H Meier, and Anja Böckmann

This manuscript has been accepted after peer review and appears as an Accepted Article online prior to editing, proofing, and formal publication of the final Version of Record (VoR). This work is currently citable by using the Digital Object Identifier (DOI) given below. The VoR will be published online in Early View as soon as possible and may be different to this Accepted Article as a result of editing. Readers should obtain the VoR from the journal website shown below when it is published to ensure accuracy of information. The authors are responsible for the content of this Accepted Article.

To be cited as: ChemPhysChem 10.1002/cphc.201800211

Link to VoR: http://dx.doi.org/10.1002/cphc.201800211 


\title{
Localizing conformational hinges by NMR: where do HBV core proteins adapt for capsid assembly?
}

\author{
Lauriane Lecoq $^{[\mathrm{a}]}$, Shishan Wang ${ }^{[\mathrm{a}]}$, Thomas Wiegand ${ }^{[\mathrm{b}]}$, Stéphane Bressanelli ${ }^{[\mathrm{c}]}$, Michael Nassal ${ }^{* \mathrm{~d}]}$, \\ Beat H. Meier ${ }^{*[b]}$, Anja Böckmann*[a]
}

\begin{abstract}
The hepatitis $B$ virus (HBV) icosahedral nucleocapsid is assembled from 240 chemically identical core protein molecules and, structurally, comprises four groups of symmetrically nonequivalent subunits. We show here that this asymmetry is reflected in solidstate NMR spectra of the capsids in which peak splitting is observed for a subset of residues. We compare this information to dihedral angle variations from available $3 D$ structures, and also to computational predictions of "dynamic" domains and molecular hinges. We find that while, at the given resolution, dihedral angles variations directly obtained from the $X$-ray structures are not precise enough to be interpreted, the chemical-shift information from NMR correlates, and interestingly goes beyond, information from bioinformatics approaches. Our study reveals the high sensitivity with which NMR can detect the residues allowing the subtle conformational adaptations needed in lattice formation. Our findings are important for understanding the formation and modulation of protein assemblies in general.
\end{abstract}

When proteins assemble into larger superstructures, the constituting molecules are in many cases no longer symmetry equivalent, as they need to conform to a minimum energy configuration. This is observed in protein crystals, but also in protein fibrils or viral capsids and envelopes. In crystals, a subunit from which the entire structure can be generated by symmetry operations is called the asymmetric unit, and this concept is also used in other symmetric assemblies like icosahedra with a triangulation number $T>1$, where the monomers need to distort in nonequivalent classes of conformations upon assembly. ${ }^{[1]}$ These distortions can either be distributed over the protein chain, or be localized at a few key residues, i.e. hinges, interconnected by predominantly rigid bodies. The identification of hinge residues is important in a variety of areas, and computational software, as for example DynDom, ${ }^{[2]}$ has been developed to determine "dynamic" domains (with dynamics referring there to differences between two static structures, and not actual flexibility), hinge axes and hinge bending residues from two protein conformers, and was

[a] Dr. Lauriane Lecoq, Shishan Wang, Dr. Anja Böckmann Molecular Microbiology and Structural Biochemistry, Labex Ecofect, UMR 5086 CNRS/Université de Lyon

69367 Lyon, France

E-mail: a.bockmann@ibcp.fr

[b] Dr. Thomas Wiegand, Prof. Beat H. Meier

Physical Chemistry, ETH Zurich

8093 Zurich, Switzerland

E-mail: beme@ethz.ch

[c] Dr. Stéphane Bressanelli

Institute for Integrative Biology of the Cell (I2BC), CEA, CNRS, Univ Paris Sud, Université Paris-Saclay, 91198, Gif sur Yvette, France

[d] Prof. Michael Nassal

University Hospital Freiburg, Dept. of Medicine II / Molecular Biology,

Medical Center, University of Freiburg.

Hugstetter Straße 55. 79106 Freiburg. Germany

E-mail: michael.nassal@uniklinik-freiburg.de successfully applied to compare structures with a resolution higher than $3 \AA^{[3]}$

The HBV particle is composed of an outer envelope and an inner nucleocapsid formed by a self-assembling core protein (Cp) of $20 \mathrm{kDa}$. The first $\sim 140$ amino acids of $\mathrm{Cp}$ are sufficient for capsid assembly. ${ }^{[4,5]}$ First cryo-electron microscopy (cryoEM),${ }^{[6,7]}$ and later on crystallography, ${ }^{[8]}$ revealed the structure of Cp149, as a mainly a-helical protein which dimerizes via formation of a four-helix bundle. In the assembled capsid, these bundles appear as perpendicular dimeric spikes punctuating the capsid shell that is formed by lateral dimer-dimer contacts. The $\mathrm{T}=4$ icosahedral symmetry accommodates four distinct (A, B, C and D) environments with 60 molecules each. Two types of dimers, 60 of type $A B$ and 60 of type $C D,{ }^{[9]}$ assemble in 12 fivefold vertices composed solely by subunit $A$, and 30 quasisixfold vertices composed by subunits $B, C$ and $D$. Because the HBV capsid follows classical quasi-equivalence,$^{[1]}$ the contacts between dimers around the five- and quasi-sixfold vertices are very similar and involve the same residues. More recently, cryoEM revealed structural details of HBV capsids down to $3.5 \AA$ resolution, resulting in a similar structure to X-ray for the four molecules. ${ }^{[10]}$ EM studies of the capsid have further shown that the local structural modifications caused by the insertion of additional amino acids leads to global structural changes where hinge residues play an important role. ${ }^{[1]}$ Hinge-mediated motions may also enable the dynamic structural changes suspected to accompany viral genome maturation during HBV

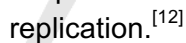

Virus capsids can also be studied by solid-state NMR. ${ }^{[13-15]}$ NMR chemical shifts, and especially those of carbon-13, are highly sensitive to backbone dihedral angles, and can detect conformational differences by chemical-shift perturbations, typically when an interactant is added, or in protein complexes (for a review see ${ }^{[16]}$ ). When different conformations (or more general crystallographically inequivalent species) are present in a single entity, this results in the observation of peak splitting in the spectra. ${ }^{[17-22]}$ We show here, at the example of the HBV capsid, that peak splitting is indeed observed in the spectra for a specific subset of residues, and that these sites correlate with molecular hinges, but also the "dynamic" domains, identified by the bioinformatics approach in DynDom.

We here investigated E. coli self-assembled Cp149 HBV capsids forming homogeneous $\mathrm{T}=4$ icosahedra with a typical diameter of $30 \pm 3 \mathrm{~nm}$ (Figure 1a). The ${ }^{13} \mathrm{C}$ and ${ }^{15} \mathrm{~N}$ resonances of the $\mathrm{Cp} 149$ protein were assigned using 3D spectroscopy, ${ }^{[23]}$ and the annotated 2D ${ }^{13} \mathrm{C}-{ }^{13} \mathrm{C}$ Dipolar Assisted Rotational Resonance $^{[24]}$ (DARR) spectrum of the capsids is displayed in Figure 1b. Sequential assignments reveal that a subset of residues experiences resonance splitting, with up to $2.5 \mathrm{ppm}$ of chemical-shift differences amongst themselves in the ${ }^{13} \mathrm{C}$ and/or ${ }^{15} \mathrm{~N}$ dimensions. Indeed, peak splitting is clearly distinguishable for residues A11, T12, L16-D22, T33-S35, L108-F110, S121W125, R127, T128, P130-Y132 and N136-P138 (Figure S1). Chemical shifts of all forms are given in Table S1. 


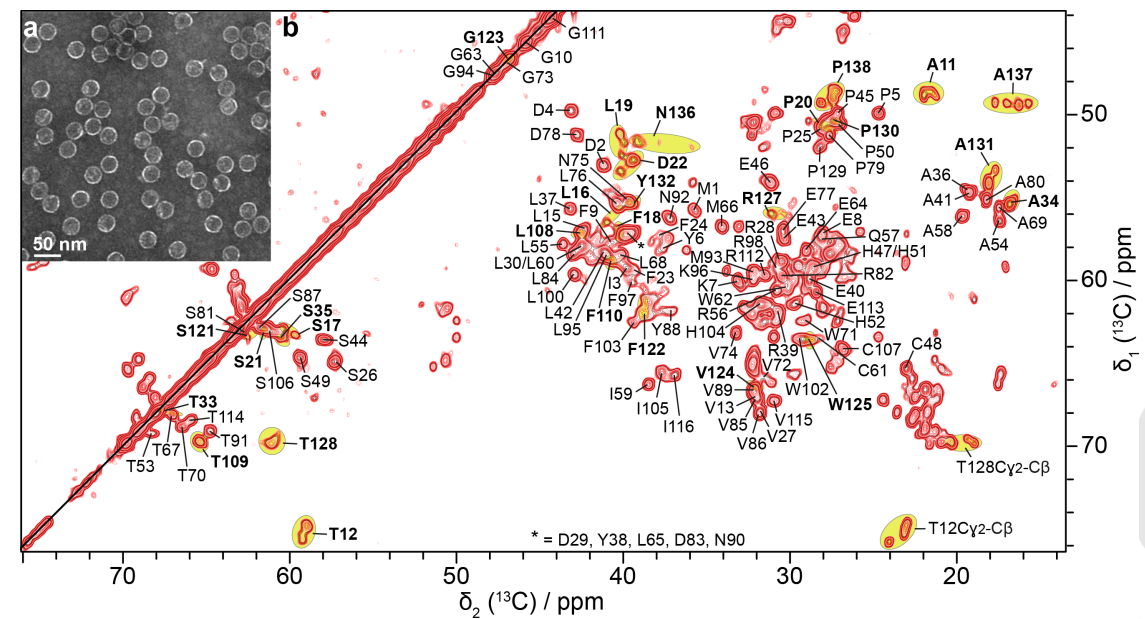

Figure 1. a) Negative-stain EM micrograph of Cp149 capsids. b) 2D ${ }^{13} \mathrm{C}-{ }^{13} \mathrm{C}$ DARR solid-state NMR spectrum of $\mathrm{Cp} 149$ capsids recorded with a mixing time of $20 \mathrm{~ms}$. All assigned $\mathrm{C} \alpha-\mathrm{C} \beta$ intraresidue correlations are labeled with the residue number except for proline residues where the $\mathrm{C}_{\gamma}$ $\mathrm{C} \delta$ peaks are labeled. Residues with peak splitting are indicated in bold letters and corresponding resonances are highlighted in yellow. The spectrum was processed using a squared cosine apodization (SSB 3).

Where four distinct signals are observed (as for example A137 shown in Figure 2a), they can be assigned to the four crystallographic distinct molecules in the asymmetric unit represented in Figure 2b. When two sets of peaks are observed, it suggests that at least one monomer is significantly distinct from the others. In total, detectable peak splitting was observed for 28 residues, representing almost $20 \%$ of the protein.

Nearly all residues showing peak splitting are located close to the fivefold (Figure 2c) and quasi-sixfold (Figure 2d) vertices, while none of the residues within the spikes of the capsids is concerned. About half of them are located in loops, where the backbone is less constrained by hydrogen bonds than in regular secondary structure elements, and the remaining are mainly located towards the beginning or end of the base plate helices (Figure S2). For A137, the four $\mathrm{C} \alpha-\mathrm{C} \beta$ correlation peaks can be easily distinguished in the $2 \mathrm{D}$ spectra (Figure 2a). An analysis of the multiple peaks from a same residue in the 2D DARR spectrum (Figure S3) and 3D spectra (Figure S4) reveals similar intensities, which suggests that the peaks indeed correspond respectively to the $60 \mathrm{~A}, \mathrm{~B}, \mathrm{C}$ and $\mathrm{D}$ subunits per capsid. The same behavior is observed for the other residues displaying peak quadrupling, namely $\mathrm{T} 12$, S35 and T128. On the contrary, when only two or three peaks are observed in the NMR spectra, one peak is usually more intense, representing 120 or 180 subunits with a rather similar environment.

The nonequivalence of the four subunits is also visible in the X-ray structure ${ }^{[8]}$ as seen in Figure $3 a$ where the four monomers are aligned using PyMOL. ${ }^{[25]}$ Some residues display significant differences between the four monomers such as Y132, N136 or A137. As a consequence, the intermolecular contacts in the five- and quasi-sixfold vertices are indeed different, as shown in Figures $3 b$ and $c$ at the example of $A 137$. Naively, one would assume that the deviation between the dihedral angles of the molecules in the unit cell would correlate with the residues showing peak splitting. An analysis of the local variations in backbone torsion angles $\varphi$ and $\psi$ between the four monomers in the X-ray structure (as standard deviations), which are shown in black in Figure 3d, however reveals that this is not the case. Instead, the variations are rather uniform along the sequence, with few $\mathrm{N}$ - and $\mathrm{C}$-terminal residues, plus some residues in the spike loop displaying higher variations. It has however already been realized that the sum of torsion-angle rotations needed to superimpose two structures to the experimental accuracy can be 10 times larger than when just adding $\varphi$ and $\psi$ differences along the protein backbone, ${ }^{[26]}$ even for structures showing higher precision than Cp149 (1.7 vs 3.5 A). Using computer programs which localize differences between two given (X-ray) structures, ${ }^{[26-28]}$ one obtains for example for two nominally identical structures of dihydrofolate reductase at $2.2 \AA$ (PDB codes $1 \mathrm{RG}^{[29]}$ and $1 \mathrm{DDS}^{[30]}$ ) a difference in dihedral angles of $9^{\circ} \pm 11^{\circ} .{ }^{[26]}$ Even between the dihedral angles obtained from two high resolution and nominally identical structures, significant dihedral angle variations $\left(4.4^{\circ}\right)$ are found (Figure S5).
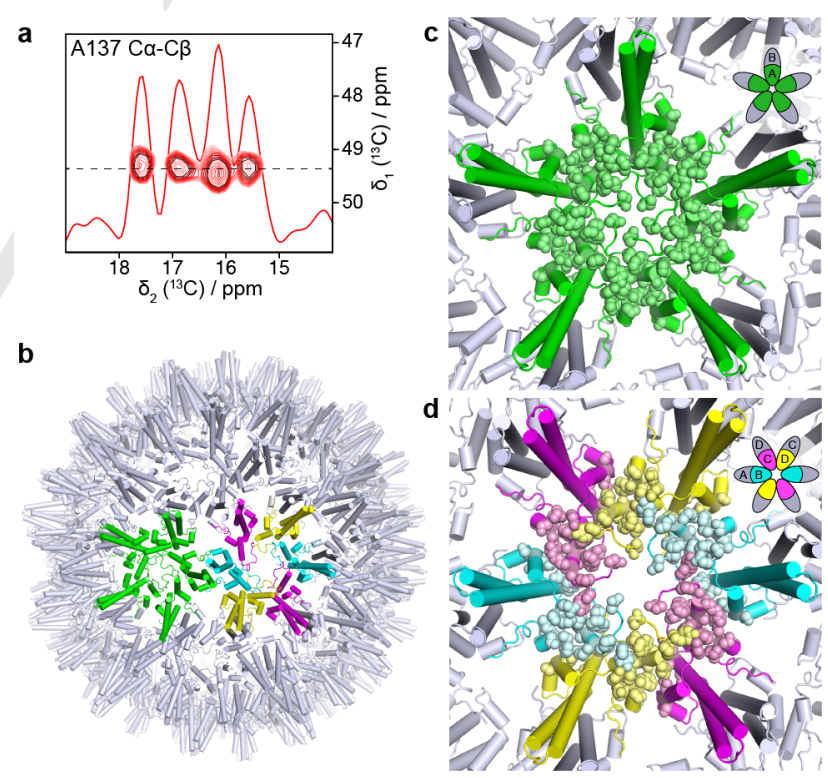

Figure 2. a) Extract from a $2 D$ DARR spectrum, as well as a $1 D$ trace, displaying the Ca-C $\beta$ correlations of Ala137 showing a peak quadrupling, corresponding to the four molecules in the unit cell. b) Capsid structure (PDB: $1 Q G T^{[8]}$ ), with localization of the monomers on the five- and quasi-sixfold vertices. c, d) Sphere representation of the side chains of the 28 residues showing NMR peak splitting, located near the fivefold (c) and quasi-sixfold (d) interfaces, shown on the capsid structure. On the top right are given the schematic representations of the subunit arrangement in the $\mathrm{T}=4$ capsid, described $\mathrm{in}^{[8]}$. A, B, C and D subunits are shown in green, cyan, magenta and yellow, respectively. The $A$ subunits form pentamers while $B, C$ and $D$ subunits form the hexamers. 

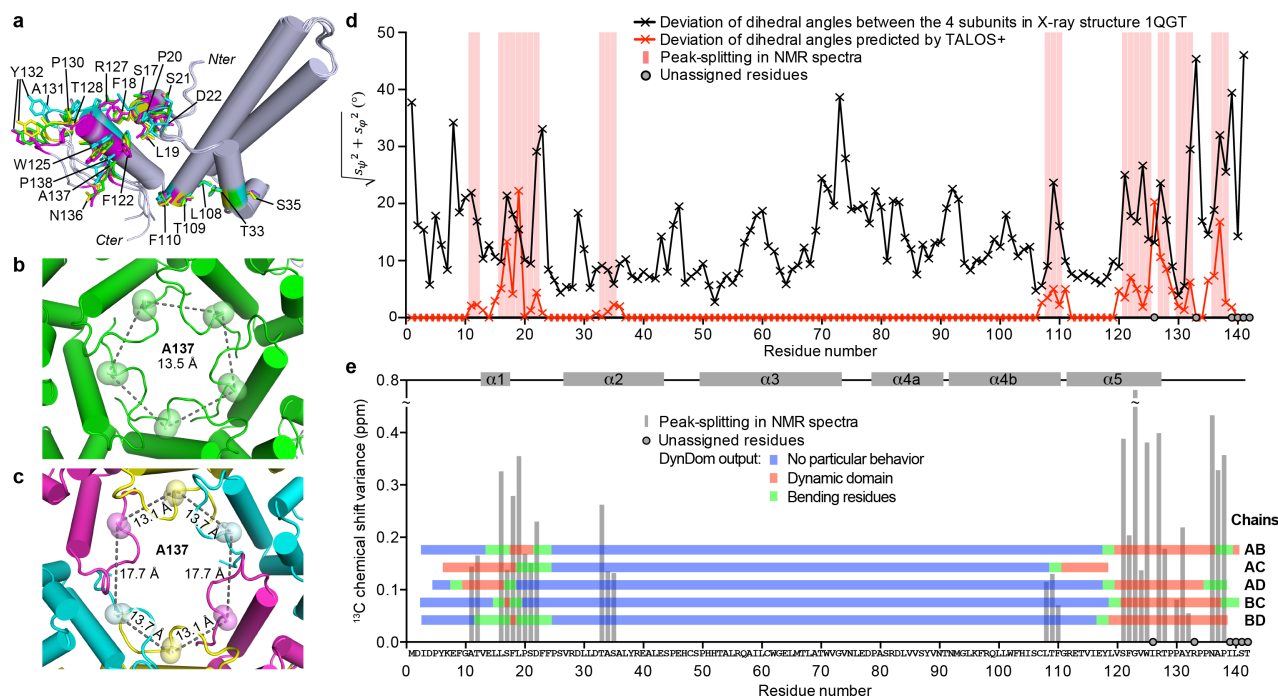

Figure 3. a) Overlay of the four capsid subunits to visualize on one hand the differences in the X-ray structures between the molecules in the asymmetric unit (PDB: $1 \mathrm{QGT}^{[8]}$ ), and on the other hand the residues for which peak splitting is detected by solid-state NMR (shown as sticks in the color of the corresponding subunit: green, cyan, magenta and yellow for $A, B, C$ and $D$ subunit respectively). b) Zoom into the five-fold vertices with equivalent distances shown for the $C \beta s$ of $A 137$ between the five A subunits. c) Zoom into the quasi-sixfold vertices with nonequivalent distances shown for the $C \beta s$ of A137 between each B, C and D subunit. d) Plots of the rms of the standard deviations between the backbone dihedral angles of the four subunits of the X-ray structure of Cp149 (PDB 1QGT ${ }^{[8]}$, black line), and the corresponding value predicted from the chemical shifts of the different forms observed by NMR using TALOS+ ${ }^{[31]}$ (red line). Residues displaying peak splitting in the NMR spectra are highlighted by pink bars. Unassigned residues are marked by grey circles. e) ${ }^{13} \mathrm{C}$ chemical shift variance of the different forms observed by NMR (grey bars) and identification of residues involved in interdomain motions using the DynDom software ${ }^{[2]}$ (horizontal bars). The "dynamic" domains (in red) and the bending residues (in green) are determined by comparing pairwise the $A, B, C$ and $D$ chains of the $X$-ray structure (PDB 1QGT ${ }^{[8]}$ ).

Backbone torsion angles can also be derived from the NMR secondary chemical shifts. Using a databank approach, the program TALOS ${ }^{[31]}$ delivers $\varphi$ and $\psi$ from the chemical shifts of the residue to be investigated, plus for one neighbor on each side. The standard deviation between the dihedral angles predicted from the split resonances (Table S1) is shown in red in Figure 3d. Evidently, the torsion-angle variations differ between the two methods: while NMR shows an average deviation of $1.5^{\circ}$ and only 38 residues involved in changes, the X-ray analysis lacks the clear pattern observed by NMR, with an average deviation of $14^{\circ}$, and no link between the two dihedral-angle variations is apparent. Given the observations described above, this is no great surprise and the variations in the X-ray structure are probably background differences. ${ }^{[26]}$ (For similar results from the comparison between the X-ray and EM capsid structures see Figure S6 and S7).

Confronting the NMR data to predictions from the program DynDom $^{[2]}$ designed to identify hinges from the comparison of two X-ray structures was more successful. DynDom defines "dynamic" domains, as well as residues involved in interdomain bending (http://fizz.cmp.uea.ac.uk/dyndom/dyndomMain.do). (While from the wording one could infer a motional analysis, the actual flexibility of the protein is of no subject in this context, as only two structures are compared.) Applied to the capsid monomers in a pairwise analysis using the DynDom webserver (Figure 3e), "dynamic" domains (in red) and bending residues (in green) were identified for all pairs (with exception of the $C$ and $D$ monomers which do not reveal any "dynamic" domains or hinges). The individual comparisons yield similar regions involved in interdomain bending, one near the $\mathrm{N}$-terminal, and another in the C-terminal domain. The overlap of the pairwise analyses is, with the exception of $\mathrm{AC}$, rather consistent. DynDom classifies the residues in three classes: rigid domains, hinges and, in addition, the "dynamic" domains which are not necessarily rigid, but show an intradomain variation smaller than the interdomain motion. For the C-terminal, DynDom clearly differentiates between "dynamic" domains (residues 121-135) and bending residues $(\sim 117-120)$, while in the $\mathrm{N}$-terminal region this distinction is less clear-cut, and globally comprises residues 8-24. Residues T109 and F110 were only detected as bending residues between subunits $A$ and $C$. The differences observed between the pairwise comparisons of monomers A-D illustrate the limit of the approach, but overall, good agreement is found. It is interesting that both the "dynamic" domain and hinge residues identified using DynDom strongly correlate with the residues for which NMR peak splitting is observed (observed chemical-shift variance shown as grey bars in Figure $3 e$ ), which reveals that actually the "dynamic" domains show a variability comparable to the hinges as response to constraints from capsid assembly. In particular, splitting in the C-terminal region for residues S121 to Y132, including helix $\alpha 5$, coincide with the "dynamic" domain from the DynDom analysis. The large chemical-shift variations observed for residues 136-138 fall into the range of the mechanical hinge predicted by DynDom. Interestingly, no peak splitting is observed for the predicted hinge residues 118-120, but only from residue 121 onwards. These residues are part of helix $\alpha 5$ spanning residues $\mathrm{R} 112-\mathrm{R} 127$, and even if the hinge seems shifted compared to the DynDom prediction, it still comprises parts of the helix. Residues A11, T12 and L16-D22 displaying peak splitting coincide with the $\mathrm{N}$-terminal region identified as "dynamic" hinges by DynDom, which however shows a rather large distribution over the different pairs, including regions not highlighted by NMR. T109 and F110, exclusively detected as bending residues between $A$ and $C$ monomers by DynDom, display, together with L108, peak splitting in the NMR spectra. Interestingly, residue G111 in this same region has been described as hinge in an early EM study. ${ }^{[11]}$ Residues T33 to S35 are only identified by NMR peak splittings, and are located in helix $\alpha 2$ which is facing helices $\alpha 1$ and $\alpha 5$, part of the $\mathrm{N}$-terminal and $\mathrm{C}$-terminal hinge regions 
respectively. Overall, these results show that the peak splitting observed in the NMR spectra correlates to a large extent with both the hinges and the "dynamic" domains identified by the DynDom method.

It has been suggested early on that the accurate recognition of sites involved in interdomain motions, here linked to the creation of adequate interfaces on mutimerization, may identify possible target sites for drugs. ${ }^{[27]}$ And indeed, in the available structures of the core protein in complex with inhibitors, ${ }^{[32,33]}$ T33, T109, F110, F122, V124, W125, T128 and Y132 residues are in close contact with small molecule assembly modulators (Figure S8), which are currently intensely investigated as potential new anti-HBV drugs. ${ }^{[34]}$ Interference with the possibility to create adequate conformers via hinge motion, or inversely the stabilization of certain conformations promoting assembly, is likely involved in assembly modulation by these drugs. Alternatively, the hinges might play a role in accommodating assembly modulators at critical locations at protein interfaces.

In summary, we have demonstrated that structural differences between quasi-equivalent HBV core proteins occur in strategic positions near the interfaces between the dimers, and no distortions are found in the helices that form the spikes of the capsid. This immediate information from NMR correlates well with information on molecular hinges extracted via bioinformatics methods, here DynDom, from high-resolution 3D structures but is more explicit because it is a direct residue-specific observation not requesting extensive postprocessing of the primary data. The site-selective information from NMR is of particular importance to further analyze in detail the different conformations which the HBV capsid is thought to access during genome and particle maturation, ${ }^{[12]}$ as well as its interactions with assembly modulators.

Acknowledgements: This work was supported by the Swiss National Science Foundation (Grant 200020_159707), the French National Research Agency (ANR) (ANR-14-CE09-0024B), by the LABEX ECOFECT (ANR-11LABX-0048) of Université de Lyon, within the program "Investissements d'Avenir" (ANR-11-IDEX-0007) operated by the ANR, the DFG grant NA154/94 and the Federal Ministry for Economic Affairs and Energy grant ZIM ZF4010301 CR5 (MN), the TGIR-RMN-THC Fr3050 CNRS, the Chinese Scientific Council (SW) and the European Marie Skłodowska-Curie program (H2020-MSCA-IF-2016 748516). BM acknowledges support from the European Research Council (ERC) under the European Union's Horizon 2020 research and innovation programme (grant agreement $n^{\circ} 741863$, FASTER). We thank the Centre d'Imagerie Quantitative Lyon-Est (CIQLE) for access to the EM platform, and Bettina Böttcher for interesting and helpful discussions. Keywords: Hepatitis B virus • Nucleocapsid • Core protein • Solid-state $\mathrm{NMR} \cdot$ Asymmetric unit
D. L. D. Caspar, A. Klug, Cold Spring Harb. Sym. 1962, 27, 1-24. D. Taylor, G. Cawley, S. Hayward, Bioinformatics 2014, 30, 3189-3196.

R. Koike, T. Amemiya, T. Horii, M. Ota, J. Struct. Biol. 2017, DOI 10.1016/j.jsb.2017.12.004.

F. Birnbaum, M. Nassal, J. Virol. 1990, 64, 3319-3330.

A. Gallina, F. Bonelli, L. Zentilin, G. Rindi, M. Muttini, G. Milanesi,

J. Virol. 1989, 63, 4645-4652.

B. Böttcher, S. A. Wynne, R. A. Crowther, Nature 1997, 386, 88 91.

J. F. Conway, N. Cheng, A. Zlotnick, P. T. Wingfield, S. J. Stahl,

A. C. Steven, Nature 1997, 386, 91-94.

S. A. Wynne, R. A. Crowther, A. G. Leslie, Mol. Cell 1999, 3, 771-780.

A. Zlotnick, N. Cheng, J. F. Conway, F. P. Booy, A. C. Steven, S.

J. Stahl, P. T. Wingfield, Biochemistry 1996, 35, 7412-7421.

X. Yu, L. Jin, J. Jih, C. Shih, Z. Hong Zhou, PLoS ONE 2013, 8, e69729-11.

B. Böttcher, M. Vogel, M. Ploss, M. Nassal, J. Mol. Biol. 2006 , 356, 812-822.

M. Nassal, Virus Res. 2008, 134, 235-249.

C. M. Quinn, M. Lu, C. L. Suiter, G. Hou, H. Zhang, T. Polenova, Prog. Nucl. Mag. Res. Sp. 2015, 86-87, 21-40.

G. Abramov, O. Morag, A. Goldbourt, J. Magn. Reson. 2015, 253 80-90.

L. B. Andreas, K. Jaudzems, J. Stanek, D. Lalli, A. Bertarello, T. Le Marchand, D. Cala-De Paepe, S. Kotelovica, I. Akopjana, B. Knott, et al., Proc. Natl. Acad. Sci. U.S.A. 2016, 113, 9187-9192. M. P. Williamson, Prog. Nucl. Mag. Res. Sp. 2013, 73, 1-16.

J. Gath, L. Bousset, B. Habenstein, R. Melki, A. Böckmann, B. H. Meier, PLoS ONE 2014, 9, e90659-11.

A. Böckmann, A. Lange, A. Galinier, S. Luca, N. Giraud, M. Juy, H. Heise, R. Montserret, F. Penin, M. Baldus, J. Biomol. NMR 2003, 27, 323-339.

H. L. Frericks Schmidt, L. J. Sperling, Y. G. Gao, B. J. Wylie, J. M. Boettcher, S. R. Wilson, C. M. Rienstra, J. Phys. Chem. B 2007, 111, 14362-14369.

P. Ma, Y. Xue, N. Coquelle, J. D. Haller, T. Yuwen, I. Ayala, O. Mikhailovskii, D. Willbold, J.-P. Colletier, N. R. Skrynnikov, et al., Nat. Commun. 2015, 6, 8361.

L. B. Andreas, M. T. Eddy, R. M. Pielak, J. Chou, R. G. Griffin, J. Am. Chem. Soc. 2010, 132, 10958-10960.

L. B. Andreas, M. Reese, M. T. Eddy, V. Gelev, Q. Z. Ni, E. A.

Miller, L. Emsley, G. Pintacuda, J. J. Chou, R. G. Griffin, J. Am. Chem. Soc. 2015, 137, 14877-14886.

L. Lecoq, S. Wang, T. Wiegand, S. Bressanelli, M. Nassal, B. H. Meier, A. Böckmann, Biomol. NMR Assign. under revision.

K. Takegoshi, S. Nakamura, T. Terao, Chem. Phys. Lett. 2001, 344, 631-637.

The PyMOL Molecular Graphics System, Version 2.0

Schrödinger, LLC, 2015.

B. K. Chapman, O. Davulcu, J. J. Skalicky, R. P. Brüschweiler, M.

S. Chapman, Struct. Fold. Des. 2015, 23, 1190-1198.

S. Hayward, H. J. Berendsen, Proteins 1998, 30, 144-154

N. Echols, D. Milburn, M. Gerstein, Nucleic Acids Res. 2003, 31, 478-482.

J. Dunbar, H. P. Yennawar, S. Banerjee, J. Luo, G. K. Farber, Protein Sci. 1997, 6, 1727-1733.

M. R. Sawaya, J. Kraut, Biochemistry 1997, 36, 586-603.

Y. Shen, F. Delaglio, G. Cornilescu, A. Bax, J. Biomol. NMR 2009, 44, 213-223.

K. Klumpp, A. M. Lam, C. Lukacs, R. Vogel, S. Ren, C. Espiritu R. Baydo, K. Atkins, J. Abendroth, G. Liao, et al., Proc. Natl. Acad. Sci. U.S.A. 2015, 112, 15196-15201.

Z. Zhou, T. Hu, X. Zhou, S. Wildum, F. Garcia-Alcalde, Z. Xu, D. Wu, Y. Mao, X. Tian, Y. Zhou, et al., Sci. Rep. 2017, 7, 42374. L.-Y. Mak, D. K.-H. Wong, W.-K. Seto, C.-L. Lai, M.-F. Yuen,

Expert Opin. Ther. Tar. 2017, DOI

10.1080/14728222.2017.1397134. 
WILEY-VCH

\section{COMMUNICATION}

The HBV capsid shows four different molecules in the $T=4$ icosahedral assembly. This results in peak splitting observed in the solid-state NMR spectrum for residues showing conformational variations between the different monomers. We show that this splitting directly locates molecular hinges allowing for capsid assembly.

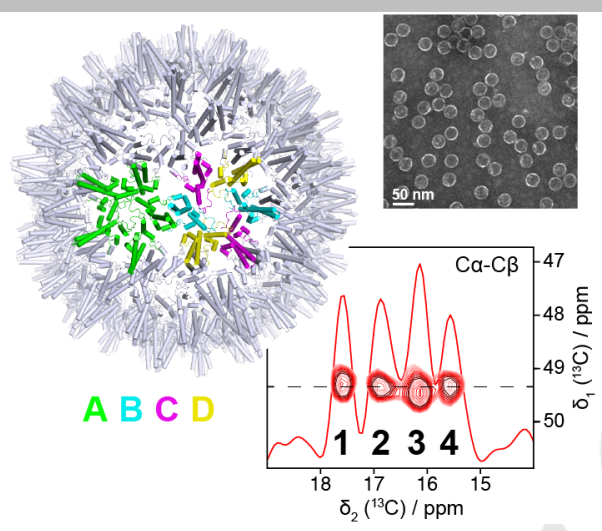

Lauriane Lecoq, Shishan Wang,

Thomas Wiegand, Stéphane

Bressanelli, Michael Nassal ${ }^{*}$, Beat $H$. Meier*, Anja Böckmann*

Page No. - Page No.

Localizing conformational hinges by NMR: where do HBV core proteins adapt for capsid assembly? 